\title{
Sense of Times and Aesthetic Characteristics of Modern Book Decoration Design
}

\author{
Dan Liu \\ Shaanxi Vocational and Technical College, Xi'an, 710100, China
}

Keywords: modern books; decoration design; sense of times; aesthetic characteristics

\begin{abstract}
The modern book decoration design is a comprehensive category of art, which is an important part in the books and expresses the creation connotation of books in an artistic form, and strengthen the original artistic flavor in the books through the modern decoration design, making the readers can feel the strong artistic atmosphere while reading the books. Therefore, the modern book decoration design has a strong sense of times and some distinctive aesthetic characteristics, so as to express the strong artistic vitality and the characteristics of keeping up with the times.
\end{abstract}

\section{Introduction}

The modern book decoration design, subordinated to the books, mainly means design the appearance, materials, layout and making involved in the process of book production. Today, there are still people understanding it as the cover graphic design partially, actually, the cover graphic design is only a part of the book decoration design, because it is a kind of comprehensively art, can accurately reflect the internal spirit of the original works and is widely communicated in the artistic form, with an independent artistic form, belonging to the scope of spiritual commodity. If the books are lack of the link of book decoration design, their influence on and affection to readers will be influenced, because book decoration design reflects certain characteristics of times and the current people's different aesthetic demands, with different aesthetic characteristics, and it will require the book decoration designers to be able to carry out innovative design according to different requirements of times and people's aesthetic demands, so as to meet the better meet the readers' demands.

\section{Book and book decoration design}

Book belongs to a kind of systematic comprehensive artistic engineering, which changes with the times, and is a marginal science integrating multiple categories of disciplines. Books record the development history and civilization of a country. China is a county of ancient civilization with thousands of years' splendid culture, and books even have an indelible influence, which have a close link with the four great inventions in ancient China, especially the papermaking technology and printing technology. The Chinese books have a high status that cannot be ignored in the world book decoration design history. The modern Chinese books are developed from the blending of a lot of national elements based on the ancient books, with the world characteristics.

Then, as an important part of the human life, what is the book decoration design on earth? According to the basic meaning of design, book decoration design means to conceive and plan a book, so as to lay a good foundation for its formal publication. It should be said that the book decoration design is a comprehensive art, which is different from other artistic expression forms, and it can be seen by the readers really through printing and publishing only after completion of design. The book decoration design must conform to the original contents of the books, reflect the original ideological contents of the books, and also need to be appropriately designed and bound to form books. It should be said that the topic selection of book decoration and artistic and literature works are diversified, therefore, the decoration design techniques are also diversified, which emphasize the diversification characteristics of the design style, and appropriate design techniques are designed in order to better reflect different ideological contents in the books, so as to express the original contents more appropriately, and this requires the book decoration designers can have a 
skillful design conception, and requires that the book decoration design should be scientific, artistic and uniform, the protective cover (including enveloping cover), cover or flyleaf, texts and illustrations as well as copyright page should fully reflect the consistency between contents and form.

\section{Sense of times of modern boom decoration design}

The book decoration design must cater to the requirements of development of times, therefore, there must be a strong sense of times in its design process. Then, the sense of times of modern book decoration design are reflected in the following:

(1) Having a profound cultural deposit. In the modern book decoration design, it is required to fully reflect the content essence of the original books and make readers experience the soul of the books through their own vision and contact. If the book decoration design is only the simple and plain graphic design, the spiritual expression level of the books will be greatly reduced. Therefore, the book decoration designer should deeply understand and accurately refine the contents and connotation of the books before design of the books, especially it is required to fully mine the cultural elements in the books and apply them in some illustrations and texts or express them from the appearance of special materials. With the change and development of the times, the readers' reading concept is also being updated continuously, which do not only require that the books have a good visual effect, but also have good contact feeling and protect the eyesight etc., while these design requirements also make the book decoration design have higher requirements, i.e. the book decoration design should realize the self-innovation and change from internally to externally, require designers to fully mine the connotation of the books, deeply the understand the readers' psychological demand, and reasonably arrange and plan the book decoration design, so that the spiritual connotation of books can be expressed through many means. The original book decoration design copied the foreign colleagues mechanically, and later, influenced by the international culture, more unique design styles have been pursued, reflecting the unique cultural flavor;

(2) Innovation of book form. In the modern book decoration design, the form structure of the book cover is often a link that needs to be considered most, because different structural forms of the books will bring different feelings and connotations to the readers. Generally, the vertical structural form can make the books present lofty, while the transverse one purists a more stable visual effect. Of course, there are also many other structural forms with different visual effect, making the cover of the books have various expression forms. The book decoration design constitutes important book decoration design elements through different linear expressions, black and white comparison and interaction and different symbolic meanings, making the book decoration design art penetrate various visual fields of the books through various many expression forms, so that the readers can enjoy the beautiful moments while reading the books and turning the pages. Then, this requires the book designers to give independent forms to the book cover, back cover, texts and layout etc. in the process of decoration, and make them blended with the external form of the books, so that the book decoration design will present unique artistic charm in the overall design of books. In the modern book decoration design, the breakthrough and innovation in structural form are mainly to make the book decoration design present different senses of space and visual effect through the coordination and comparison of points, lines, planes, colors and other elements, so that the book decoration design will shock people's heart through the formal meanings.

(3) Overall design of book decoration. The modern book decoration design is a comprehensive project, which requires that the decoration designers have different abilities, and requires that they can comprehensively master the whole book, and overall consider and design the book contents on the basis of mastering the overall contents of the books. The book decoration designers should monitor and design the materials format and making methods of the books, and let each design link generate a good visual effect to the readers. Therefore, we say that the book decoration design has become a multi-level 3D systematical process in the times background of modernization, and the book decoration designers should master the overall style from macro aspects. They must timely update their design concepts, adapt to the demands of development of times, cater to the demand of 
development of market economy and make the book decoration design form a relatively effective design system. Take the book design work Elegant Suzhou Folded Fan by the Chinese famous book decoration designer Lyu Jingren as an example, its decoration design style keeps highly consistent with the topic of the original books, making the whole book seem elegant and meticulous without losing its gorgeousness, which cannot make the readers experience the scientific, prudent and skillful design arrangement while reading the book, but can also make them feel an appropriate reading rhythm and obtain joyful reading experience. This is the concentrated reflection of the designers' good humanistic quality, and also reflection to overall control the book decoration design.

\section{Aesthetic characteristics of modern book decoration design}

Since we have know that the modern book decoration design have a strong sense of times, the designers are required to have macro design awareness and comprehensive design ability. So we should also fully mine the unique language and design conception in the modern book decoration design, which centrally reflect the aesthetic styles and characteristics of the book decoration design. In fact, in the current society, people's aesthetic demands have been more and more complicated and diversified, and the book decoration design technique dominated by the practical function previously has been unable to meet the increasing demands of aesthetic taste, and people emphasize the aesthetic value of the books while concerning the practical function of the books. It should be said that the modern book decoration design makes the book obtain completely new vitality through many aesthetic characteristics. Therefore, we will explain the aesthetic characteristics in detail. Generally, the modern book decoration design mainly includes the following aesthetic characteristics:

(1) Integrated characteristics. As the most popular design concept in the current international book decoration design field, the integration emphasize that the book decoration design must be an overall concept and system, requiring that the cover, spine, layout design and other pats in the book decoration design keep highly consistent, especially to make the readers obtain the enjoying of beauty. Like stated by the "most beautiful book" comment committee of Europe, the most beautiful books should have appropriate typeface, flyleaf and appendix and beautiful layout design, so that the whole book seem to be a harmonious and beautify unity, and makes the readers have a feeling of convenience while using the book. In the whole book decoration design, the cover design is the most important and essential, which first influences people's visual experience, and then directly influences people's initial understanding and feeling to the books. Therefore, the cover of the books should serve the contents and keep consistent with the design in other aspects. In addition, the spine, layout design and illustrations should also give people enjoying of beauty;

(2) National characteristics. Books are important means to record a state and nation, so during the boo decoration design, in addition to having certain modern sense, it is also required to design the books in combination with the state and national characteristics. It should be said that in the current times, the book decoration design must be blended with certain national cultural spirits, find out the unique cultural aesthetic expressions of the nation, and make the book design present unique artistic style. Then, specifically in China, in the modern book decoration design, it is required to blend the traditional Chinese styles and Chinese cultural and artistic spirits into the design, emphasize the pure and lofty, simple and elegant aesthetic characteristics, represent the original Chinese national elements and make readers feel a mysterious oriental flavor while feeling a shocking modern sense;

(3) Characteristics of beautiful materials. The book materials mean the texture of the materials used for the books, belonging to an appearance phenomenon that the readers can see. Here the materials are not the simple materials, but are improved to the height of art by decoration design means, and are endowed with rich connotations and meanings, making the readers can appreciate the charm of the textual works while appreciating he books, and also appreciate the beauty of book materials, which is beneficial to upgrade the level of the textual works better. The materials of modern books have been developed to many advanced paper materials from the oracle bone, jade, bamboo slip, silk and paper. For example, art paper, matt coated paper and other materials with plastic properties 
like glass paper and parchment, all these make the book decoration design developed more. They require that the decoration designer can carry out design according to different materials, achieve the individualized expression of different materials and make the materials expression different artistic design effect in the hand of designers.

(4) Characteristics of strange forms. With the update and upgrading of technology and design concept, various different creative book forms have appeared to the modern books, for example, hollow books and 3D boos, in which the hollow books are to design the books hierarchically in the artistic form of virtual-real combination, which can make the readers experience the happy of imagination in the specious reading process, while the 3D books make the designer break through the traditional book design concept and model, transform the binary plane design into the ternary design, and gradually step toward the 3D design direction, with more visual expressions than the traditional graphic design. Actually, the unique 3D decoration of the modern booms have attracted a lot of readers. Generally, 3D books are basically are the children's books, teaching materials and scientific knowledge books, which can make up the shortcomings in the texts through some 3D literature, attract the children's reading interest, stimulate their reading curiosity and enhance their practical ability, so that the modern book decoration design will present strong functions in the popularity of books.

\section{Conclusion}

In a word, with the progress and development of the times, various new technologies, materials and processes have emerged, mainly the modern book decoration design have a broader design space. They require the book decoration designer to be fully aware of the characteristics of times and unique aesthetic characteristics of the design work, so as to more emphasize the sense of times and aesthetic characteristics while considering the practical functions of the books, really make the books bring spiritual enjoying to people while providing ideological enlightenment.

\section{References:}

[1] Cao Lin. Book Decoration Creation [M] Hubei: Wuhan University of Technology Press, 2004

[2] Tang Yubing, Book Decoration Design-Teaching Materials of Modern Artistic Design [M] Hunan: Central South University Press, 2005

[3] Wu Guanying, Book Decoration [M] Jilin: Jilin Fine Arts Press, 2003

[4] Yang Shuntai, Basis for Boo Decoration Design [M] Shanghai: Shanghai Far East Publishers 